\title{
Energy-Efficient Boundary-enabled Scheduling in the Downlink of Multi-Carrier Multi-Access Heterogeneous Network
}

\author{
Ting Yang, Fabien Héliot, Chuan Heng Foh \\ 5GIC, Institute for Communication Systems, University of Surrey, \\ Guildford GU2 7XH, UK, Email: \{ting.yang, f.heliot, c.foh\}@ surrey.ac.uk
}

\begin{abstract}
Future communication networks promise to provide ubiquitous high-speed services for numerous users via densely deployed small cells. They should offer good user experiences to all the users while incurring a low operational cost to the operators. User scheduling is a well-known approach to deliver good user experience, and recent works further demonstrate that it is also beneficial to improve energy efficiency (EE). However, existing EE-based scheduling schemes tend to favor users with good channel condition which lead to unfair user experiences. In this paper, we introduce a new concept of resource allocation boundary where $\mathrm{EE}$ and user fairness can be addressed simultaneously. We derive the boundary that partition in an effective manner the users into different groups. By applying an appropriate scheduling strategy to each group of users, not only users with poorer channel conditions can be served fairly, but the EE of the system can be further improved. We also provide a low-complexity energy-efficient power allocation algorithm that is designed to fully exploit the transmit power reduction capability of small cells. Simulation results show that our new scheduling scheme can improve the EE and user fairness when compared to existing approaches, i.e. by up to $63 \%$ and $56 \%$, respectively.
\end{abstract}

Index Terms-Energy efficiency, user scheduling, user fairness, small cells, heterogeneous networks.

\section{INTRODUCTION}

One of the key aspects of the next generation of communication network [1], [2] is the deployment/integration of different types of access points, e.g. small cells (SCs), on a massive scale, a.k.a densification. This densification process is currently stimulated by mobile users craving for higher data rate. Indeed, given the soaring numbers of deployed base stations (BSs), the economic and environmental sustainability of future systems can only be ensured by reducing BS energy consumption [3]. In this regard, deploying smaller BSs with reduced coverage size and, hence, lower consumed power compared to macro BSs [4], is believed to be a promising solution [5] to help with this reduction in energy consumption. As a result, a surge of interest for energy-efficient/green communications and densification is currently being witnessed in the research community [6]-[9]. As it is reported in [10], since BSs account for over $80 \%$ of the electricity consumed by mobile network operators, reducing the power consumption of power-unlimited communication equipments, such as BSs (downlink), becomes as important as optimizing the power consumption of powerlimited equipments, e.g. mobile devices (uplink).
Multi-carrier multiple access schemes, such as orthogonal frequency-division multiplexing (OFDM)-based multiple access, provide a flexible framework for allowing numerous users to communicate at the same time, by efficiently allocating/scheduling the system resources to these users. Whereas scheduling is an effective technique for making the most out of this framework and improving the overall system performance; indeed, the way in which the resources are allocated to the users changes the distribution of the interference, which directly affects the system performance. Scheduling/resource allocation has mainly focused in the past on improving the spectrum efficiency (SE) or peak rate performance of communication system [11]. Given the growing importance of energy efficiency (EE) in mobile networks, EEbased scheduling/resource allocation (a.k.a. green scheduling) has recently attracted a lot of research interests. For instance, in a single cell context, a low-complexity energy-efficient resource allocation scheme has been designed in [12] for the downlink of an OFDM/OFDMA system. Whereas in the multi-cell context, it has been shown in [13]-[15] that multi-site cooperation and coordination can be beneficial to mitigate interference and, in turns, improve EE performance. As far as the heterogeneous network (HetNet) scenario is considered, green scheduling schemes have been developed in [16]-[18] when assuming an overlay deployment. SCs are usually deployed in either an underlay or overlay manner, i.e. operating either on the same frequency band as macro cells (i.e. co-channel deployment, frequency reuse between the tiers) or on a different band (i.e. orthogonal deployment, no frequency reuse between the tiers), respectively. As such, overlay HetNet can be viewed as two separate single-tier networks, where scheduling is applied independently on each tier; this in essence simplifies the scheduling design but, unfortunately, does not enable the system to leverage on frequency reuse for improving its performance. Overall, most of the existing green scheduling schemes, e.g. [12], [13], [15], [17], [18], are greedy scheduling schemes. They tend to favor the users with the best channel conditions given that these users achieve better EE compared to users with poor channel conditions for the same amount of allocated power [19]. This kind of scheduling, which is not particularly fair, leaves some of the users unserved, which is at odds with the future network objectives [1].

In mobile networks, user fairness is usually addressed by using either short-term management (e.g. round robin 
(RR) [20], proportional fairness (PF) [21] scheduling) or longterm planning (e.g. fractional frequency reuse (FFR) [22]) approaches. For instance, in traditional frequency planning approaches, users are categorized into inner area and celledge users based on a fixed allocation boundary [22]-[24] Fairness for the cell-edge users is achieved by avoiding interference; indeed, inner area users as well as cell-edge users in neighboring cells are allocated with different frequency resources than the ones used for cell-edge users. Although such approaches are widely used for enhancing the cell edge performance of classic cellular layout [22]-[24], they rely on static boundaries and, as such, lack of flexibility for insuring fairness and at the same time high SE or EE performance in more complex layout, e.g. HetNet layout. In the same vein, authors in [25] formulate the joint cell-center boundary selection for FFR, scheduling, and power allocation problems in a two-tier HetNet system. However, they focus on tuning the power parameters of each cell to mitigate the interference which in turns maximize the EE. Simple constant power allocation is then adopted for allocating the powers across all subchannels. One possible way to introduce more flexibility in boundary-based resource management approaches and improve the trade-off between intrinsic performance and user fairness is to integrate the boundary concept into short-term management instead of long-term planning, as we have started to investigate in [26].

This paper focuses on the design of a novel green scheduling framework for improving both the EE and user fairness in the underlay HetNet scenario. Contrary to most of the existing green scheduling works on HetNet [16]-[18], we consider here the underlay HetNet scenario, which is a more challenging scenario in terms of scheduling than the overlay scenario. Moreover, contrary to most of the existing green scheduling schemes [12]-[15], we design our novel scheduling algorithm with fairness in mind, by applying and tailoring the concept of allocation boundary (from frequency planning) to green scheduling. A concept that is used in frequency planning for improving the throughput of cell edge users and, hence, is inherently designed to improve the user fairness. The idea of user grouping can also be applied to different areas of communications. EE can be improved by exploiting new frameworks such as device-to-device communications [27], [28]. For instance, in [27], users are classified into two groups, i.e. delay-sensitive and delay-tolerant groups, where the users in the delay-tolerant group can use device-to-device communication instead of the traditional cellular network to lower their power consumption and improve their EE. The framework of our novel scheduling scheme involves two parts, in the first part, we derive an EE-based allocation boundary for the macro tier which dynamically categorizes users into inner area and cell-edge users; cell-edge users allocation is exclusive (as in OFDMA), whereas in the inner area, more than one users can be allocated on a resource element (as in multi-user superposition transmission [29]). Our dynamic allocation boundary (i.e. scheduling boundary) is updated at every scheduling interval such that it can capture the time-varying characteristics of the mobile networks, contrary to the fixed boundaries used in long-term planning schemes. In the second part, we design a novel boundary-enabled green scheduling scheme along with a low-complexity energy-efficient power allocation algorithm to coordinate the macro and underlay SCs and fully exploit the transmit power reduction capability of SCs. Note that a preliminary version of this work is available in [26]; contrary to [26], we consider here the HetNet scenario instead of the classic cellular scenario, and we derive a dynamic EE-based allocation boundary instead of an SE-based one. In addition, we develop here a bespoke energy-efficient power allocation algorithm for the HetNet scenario.

The rest of the paper is organized as follows. Section II describes the downlink underlay HetNet EE framework, i.e. the system layout and power models, which is then used to formulate our energy-based objective function, i.e. by considering the Joule-per-bit metric. In Section III, we propose our novel dynamic EE-based allocation boundary, explain the conditions of its existence, and provide its lower-bound for a specific case. In Section IV, we integrate our dynamic allocation boundary in the design of our scheduler and then derive a low-complexity energy-efficient power allocation scheme that jointly improves the user fairness and EE, by coordinating both macro and SCs transmissions. We finally compare our novel boundary-enabled green scheduling algorithm with existing green scheduling algorithm in the HetNet scenario. Our results confirm that our boundary-enabled green scheduling scheme can improve the EE by up to $63 \%$ while, at the same time, improving the fairness by up to $56 \%$.

\section{Energy EfFicient HetNet Framework}

\section{A. System Model}

We consider the downlink of a planar HetNet system, as it is depicted in Fig. 1, where sectorized macro and small BSs allow user equipments (UEs) to communicate via a multicarrier multiple access scheme over a frequency-selective and block faded channel. The macro and small BSs share the same frequency resources, which corresponds to an underlay HetNet deployment. Each macro BS serves three sectors with one directional antenna per sector, and each small BS is equipped with one omni-directional antenna to serve the UEs within its coverage area. Whereas each UE is equipped with one omni-directional antenna and can be served by either macro or small BS according to its current position. Moreover, we assume that all the BSs can coordinate their transmission, by exchanging information about their respective UEs' channel gains. More information about the coordination process can be found in [30]-[32]. Without loss of generality, we focus in the following on a representative cluster (i.e. a dodecagonal area) that is composed of three sectors; each sector is served by a different macro BS, within which, SCs are uniformly distributed. Meanwhile, UEs of each sector are served by either a macro BS or a small BS, as it is illustrated in Fig. 1. Since we only consider one sector for each macro BS, for the simplicity of the introduction, the "sectors" may also be sometimes referred to as "macro cell" in the following. This is because directional antenna is used, one BS is equipped with three antennas, each covers a 120-degree sector area, one of the sectors is just the repetition of the other two. Besides, they can be easily added up to represent a conventional cell. 


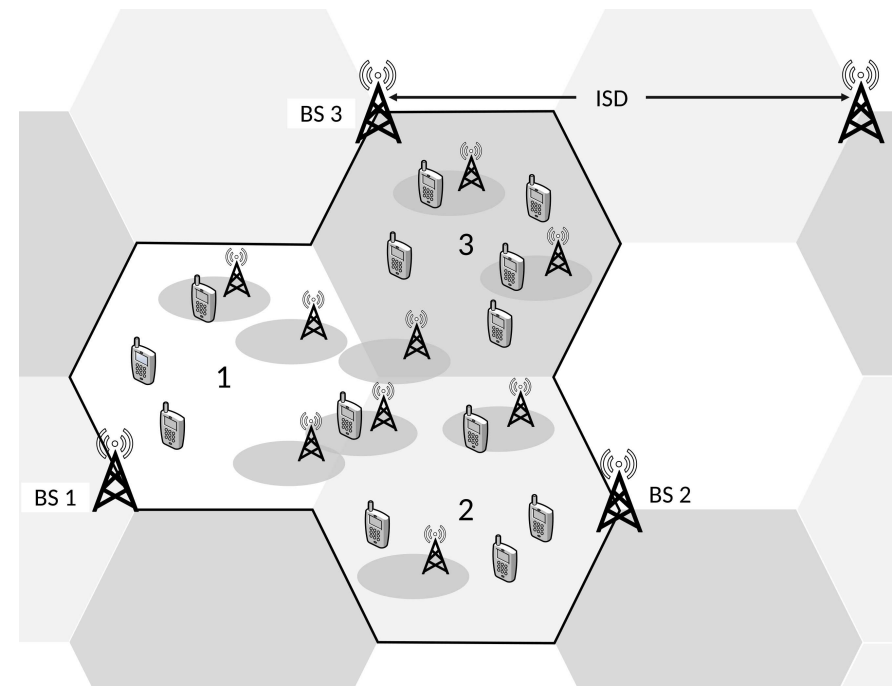

Fig. 1. Heterogeneous network layout.

Assuming a total number of $M$ BSs (macro+small BSs), that each sector has $K$ active users, that $S$ subchannels are available for scheduling these users at each time interval $t$, and that accurate channel state information (CSI) is available at both BS and UE ends (for all the links within a cluster), the channel capacity of the $k$-th user of BS $m$, i.e. served by BS $m$, on subcarrier $s$ can be expressed as [33]

$$
\mathcal{C}_{k(m)}^{s}[t]=\alpha_{k(m)}^{s}[t] B \log _{2}\left(1+\frac{g_{m, k(m)}^{s}[t] p_{m}^{s}[t]}{\sigma^{2}+I_{m, k(m)}^{s}[t]}\right) .
$$

In (1), $g_{m, k(m)}^{s}[t]$ is the channel gain between the $m$-th BS and the $k$-th user of BS $m$ over the $s$-th subcarrier, $B$ is the bandwidth of each subcarrier, $p_{m}^{s}[t]$ is the transmit power of the $s$-th subcarrier of BS $m$ and $\sigma^{2}$ is the noise power. Moreover, $\alpha$ is the subcarrier allocation indicator such that $\alpha_{k(m)}^{s}[t] \in\{0,1\}, \alpha_{k(m)}^{s}[t]=1$ if the $k$-th user of BS $m$ is assigned on subcarrier $s$ or $\alpha_{k(m)}^{s}[t]=0$, otherwise. In addition, $I_{m, k(m)}^{s}[t]$ represents the interference from the non-serving BSs over the subcarrier $s$ such that

$$
I_{m, k(m)}^{s}[t]=\sum_{\substack{j=1 \\ j \neq m}}^{M} p_{j}^{s}[t] g_{j, k(m)}^{s}[t]
$$

in the general case, $g_{j, k(m)}^{s}[t]$ is the channel gain between the $j$-th BS and the $k$-th user of BS $m$ over the $s$-th subcarrier (i.e. $j$ represents the index of non-serving $\mathrm{BS}$ ); whereas in the special case of exclusive (orthogonal) allocation, i.e. at most one user of any BSs is allocated per-subcarrier, $I_{m, k(m)}^{s}[t]=0$.

\section{B. Power Model and EE Metric}

According to [34], the energy consumption per bit, $E_{b}$, or EE $\left(1 / E_{b}\right)$, can simply be defined as a ratio between the consumed power and the rate. In order to model $E_{b}$, we first need to model the power consumption of the HetNet system in Fig 1. Although BSs can have different sizes, their power consumptions can be modeled in a similar way (transmit plus circuit powers) according to [4]. In our scenario, given that each BS has only one antenna, the total power consumption within a cluster, at time instance $t$, can be expressed as a sum of the total transmit and circuit powers, as follows,

$$
P_{\Sigma}(\mathbf{P}[t])=\sum_{m=1}^{M} \Delta_{m} \sum_{s=1}^{S} p_{m}^{s}[t]+\sum_{m=1}^{M} \sum_{s=1}^{S} P_{c, m}^{s}[t],
$$

where $\mathbf{P}[t]=\left[p_{1}^{1}[t], \ldots, p_{1}^{S}[t], p_{2}^{1}[t], \ldots, p_{M}^{S}[t]\right] \succeq 0, \Delta_{m}$ accounts for the power amplifier inefficiency of BS $m$ and $P_{c, m}^{s}[t]$ accounts for the fixed power of BS $m$ on subcarrier $s$. Note that for each BS, the total transmit power over the subcarriers should not exceed the maximum transmit power per antenna $P_{m}^{\max }$.

According to [34], the energy consumption, $E_{b}$ (1/EE) is defined as the ratio of the total consumed power to the sum-rate. In a scheduling context, where resources are allocated over time and frequency, the $E_{b}$ over the past $T$ time intervals can be formulated as

$E_{b}(\mathbf{P}, \boldsymbol{\alpha})=\frac{\sum_{t=1}^{T} \sum_{m=1}^{M} \Delta_{m} \sum_{s=1}^{S} p_{m}^{s}[t]+\sum_{m=1}^{M} \sum_{s=1}^{S} P_{c, m}^{s}[t]}{\sum_{t=1}^{T} \sum_{m=1}^{M} \sum_{s=1}^{S} \mathcal{C}_{k(m)}^{s}[t]}$,

where $\mathbf{P}=[\mathbf{P}[1], \ldots, \mathbf{P}[T]]$, and $\boldsymbol{\alpha}=[\boldsymbol{\alpha}[1], \ldots, \boldsymbol{\alpha}[T]], \boldsymbol{\alpha}[t]=$ $\left[\alpha_{1}^{1}[t], \ldots, \alpha_{1}^{S}[t], \alpha_{2}^{1}[t], \ldots, \alpha_{M}^{S}[t]\right]$.

\section{Dynamic EE-BASed Allocation Boundary}

\section{A. Theoretical Framework for the Dynamic Allocation Bound- ary}

In order to improve both the $\mathrm{EE}$ and user fairness in coordinated cellular system, we introduce in this paper the concept of dynamic EE-based allocation boundary. As already mentioned, the idea of allocation boundary is inspired by FFR where users, based on their location/channel quality relative to their serving BS, are divided into different groups and provided with different frequency resources. This helps to improve user fairness and maintain other key system performances [35], [36]. FFR, which is a long-term planning technique, uses static boundaries; whereas we develop here a dynamic boundary that can be integrated in short-term resource management (i.e. scheduling). We define our dynamic allocation boundary as a collection of points where coordinated and orthogonal resource allocation approaches achieve the same instantaneous EE performance. Coordinated allocation [32] refers to the scenario where coordination amongst BSs is used to schedule more than one user on a particular subcarrier (as in multi-user superposition transmissions); whereas in orthogonal allocation, at most one user per sub-carrier (amongst all the BSs) is allocated at a time. Given that the downlink interference for each macro UE mostly comes from its nearest nonserving macro BSs (i.e. dominant interfering BSs), without loss of generality, we focus on deriving our dynamic EE-based allocation boundary considering only two interfering macro BSs for the three-sector layout of Fig. 2. In order to derive this boundary, we have relied on the following theoretical assumptions and notations that are detailed in Table I:

- We consider the three-sector layout depicted in Fig. 2, where BS 1 , which acts as a reference point $(0,0)$, serves 
a given UE with known polar coordinate $(d, \theta)$. Similarly, BS 2 and BS 3 serve each a given user in sector 2 and 3 , respectively (which are also depicted in Fig. 2); each of these users has the same relative position to their BSs as the user in sector 1 as to BS 1 . In other words, we assume that the UEs of the three sectors (one UE per sector) have the same relative position to their serving and non serving BSs [15] (symmetric positioning).

- We assume that each BS uses the same transmit power $p$ on a given subcarrier $s$ (if a user of each given BS is allocated to this subcarrier). Note that the value of $p$ is not necessarily the same for each subcarrier. In turn, (2) is equivalent to $I_{m}^{s}[t]=p G_{m}^{s}[t]$ in the coordinated case, with $G_{m}^{s}[t]=\sum_{j=1, j \neq m}^{M} g_{j}^{s}[t], M=M_{1}$ for macro-tier.

- Given the symmetrical positioning of the UEs (see first assumption), we also assume that they experience the same desired and undesired channel gains, such that $g_{m}^{s}[t]=$ $g(d)$ and $G_{m}^{s}[t]=G(d, \theta)$.

- Among the three types of fading encompassed in $g$ and $G$, the pathloss alone can well capture the characteristic of a channel (dominant term); we therefore omit the shadowing and small-scale fading such that $g(d)=d^{-n}$ and $G(d, \theta)=d_{\text {interf1 }}^{-n}+d_{\text {interf2 }}^{-n}$, where $n$ is the pathloss exponent, $d_{\text {interf1 }}=\operatorname{ISD} \sqrt{\left(\bar{d}-\sin \left(\theta+\frac{\pi}{6}\right)\right)^{2}+\cos \left(\theta+\frac{\pi}{6}\right)^{2}}$ and $d_{\text {interf } 2}=\operatorname{ISD} \sqrt{(\bar{d}-\cos (\theta))^{2}+\sin (\theta)^{2}}$. In addition, ISD is the inter-site distance and $\bar{d}=d /$ ISD is the normalized distance.

TABLE I

TABLE OF NOTATIONS USED FOR DERIVING THE DYNAMIC SCHEDULING BOUNDARY.

\begin{tabular}{|c|c|}
\hline Notation & Definition \\
\hline$d$ & $d$ is the distance between a UE and its serving BS \\
\hline$\theta$ & $\theta$ is the angle between a UE and its serving BS \\
\hline$n$ & $n$ is the pathloss exponent \\
\hline$p_{m}^{s}[t]$ & $\begin{array}{l}p_{m}^{s}[t] \text { is the allocated transmit power on subcarrier } s \text { by BS } \\
m, p_{m}^{s}[t]=p \text { for each BS }\end{array}$ \\
\hline ISD & ISD is the inter-site-distance of the macro BSs \\
\hline $\bar{d}$ & $\bar{d}$ is the normalised distance, $\bar{d}=d /$ ISD \\
\hline$g_{m}^{s}[t]$ & $\begin{array}{l}g_{m}^{s}[t] \text { is the channel coefficient of the serving BS } m \text { to UE } \\
\text { link on subcarrier } s, g_{m}^{s}[t]=g(d) \text { for users in the same } \\
\text { group (symmetric structure) }\end{array}$ \\
\hline$G_{m}^{s}[t]$ & $\begin{array}{l}G_{m}^{s}[t] \text { is the sum of channel coefficients of the non-serving } \\
\text { BSs to UE links of BS } m \text { on subcarrier } s, G_{m}^{s}[t]=G(d, \theta) \\
\text { for users in the same group (symmetric structure) }\end{array}$ \\
\hline$P_{c, m}^{s}$ & $P_{c, m}^{s}$ is the fixed power on each subcarrier \\
\hline$A$ & $A=\frac{p}{\sigma^{2} \mathrm{ISD}^{n}}$ \\
\hline$a$ & $a=\sin \left(\theta+\frac{\pi}{6}\right)$ \\
\hline$b$ & $b=\cos \left(\theta+\frac{\pi}{6}\right)$ \\
\hline$c$ & $c=\cos (\theta)$ \\
\hline$e$ & $e=\sin (\theta)$ \\
\hline$d_{\text {interf1 }}$ & $\begin{array}{l}d_{\text {interf1 } 1} \text { is the distance from BS } 2 \text { (interfering BS) } \\
\text { served by BS } 1, d_{\text {interf1 }}=\operatorname{ISD} \sqrt{(\bar{d}-a)^{2}+b^{2}}\end{array}$ \\
\hline$d_{\text {interf2 }}$ & $\begin{array}{l}d_{\text {interf2 } 2} \text { is the distance from BS } 3 \text { (the other interfering BS) } \\
\text { to the UE served BS } 1, d_{\text {interf2 }}=\operatorname{ISD} \sqrt{(\bar{d}-c)^{2}+e^{2}}\end{array}$ \\
\hline $\bar{g}(\bar{d})$ & $\bar{g}(\bar{d})=\frac{p g(d)}{\sigma^{2}}=A \bar{d}^{-n}$ is distance-normalized received SNR \\
\hline $\bar{G}(\bar{d}, \theta)$ & $\begin{array}{l}\bar{G}(\bar{d})=\frac{p G(d, \theta)}{\sigma^{2}}=A\left(\frac{1}{\left[(\bar{d}-a)^{2}+b^{2}\right]^{\frac{n}{2}}}+\frac{1}{\left[(\bar{d}-c)^{2}+e^{2}\right]^{\frac{n}{2}}}\right) \\
\text { is the distance-normalized received interference }\end{array}$ \\
\hline
\end{tabular}

According to (4) (for a single subcarrier), the above assump-

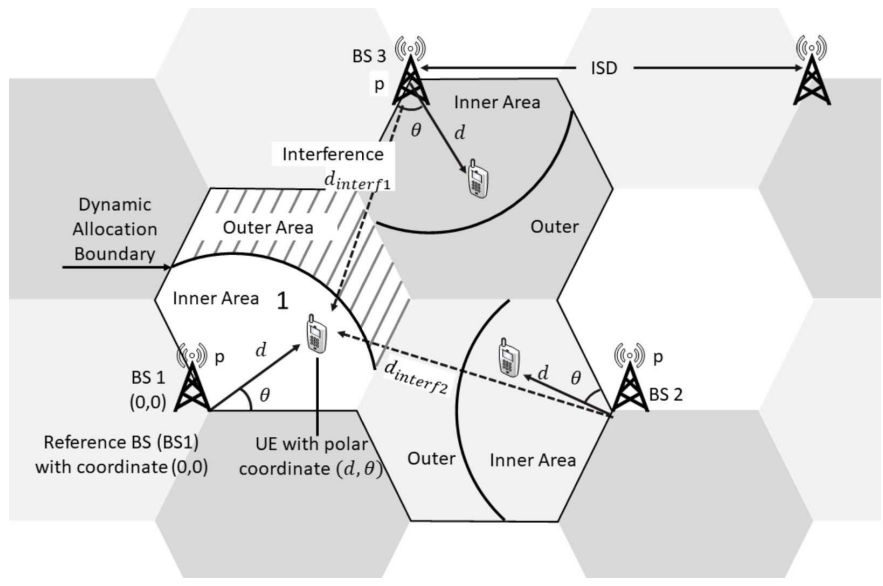

Fig. 2. Sectorized planar cellular system layout with allocation boundary.

tions, and based on [14], the per-subcarrier $E_{b}$, on any given subcarrier $s$, can be formulated as

$$
\begin{gathered}
E_{b(\text { coord })}=\frac{M\left(\Delta_{m} p+P_{c, m}\right)}{M \log _{2}\left(\frac{p g(d)}{p G(d, \theta)+\sigma^{2}}\right)} \text { and } \\
E_{b(\text { orth })}=\frac{\Delta_{m} p+M P_{c, m}}{\log _{2}\left(1+\frac{p g(d)}{\sigma^{2}}\right)}
\end{gathered}
$$

in the coordinated and orthogonal allocation scenarios, respectively. Then, based on (5a) and (5b), a sector of a cell can be partitioned into two areas (see Fig. 2): the inner and outer (sector edge) areas; $E_{b(\text { coord })}<E_{b(\text { orth })}$ in the inner area, whereas $E_{b(\text { orth })}<E_{b(\text { coord })}$ in the outer area. In addition, the limit between these two areas, which we refer to as our dynamic EE-based allocation boundary, is defined as $E_{b(\text { orth })}=E_{b(\text { coord })}$, such that

$$
\log _{2}\left(1+\frac{p g(d)}{\sigma^{2}}\right)=\beta \log _{2}\left(1+\frac{p g(d)}{p G(d, \theta)+\sigma^{2}}\right)
$$

according to (5), and where

$$
\beta=\frac{\Delta p+M P_{c, m}}{\Delta p+P_{c, m}} .
$$

Considering that $p, \sigma^{2}$, and $\beta$ are known parameters (which are all non-negative), equation (6) can be reformulated as a function of $\bar{d}$ (normalized $d$, i.e. $\bar{d}=d / \mathrm{ISD}, \bar{d} \in[0,0.5]$ ) and $\theta(\theta \in[0,2 \pi / 3])$, as

$$
f(\bar{d}, \theta, \beta)=1+\bar{G}(\bar{d}, \theta)-h(\bar{d}, \beta),
$$

where

$$
\bar{G}(\bar{d}, \theta)=A\left(\frac{1}{\left[(\bar{d}-a)^{2}+b^{2}\right]^{\frac{n}{2}}}+\frac{1}{\left[(\bar{d}-c)^{2}+e^{2}\right]^{\frac{n}{2}}}\right)
$$

and

$$
h(\bar{d}, \beta)=\frac{\bar{g}(\bar{d})}{(1+\bar{g}(\bar{d}))^{\frac{1}{\beta}}-1} .
$$

In addition, $A=\frac{p}{\sigma^{2} \operatorname{ISD}^{n}}, a=\sin \left(\theta+\frac{\pi}{6}\right), b=\cos \left(\theta+\frac{\pi}{6}\right), c=$ $\cos (\theta)$, and $e=\sin \theta, \bar{G}(\bar{d}, \theta)=\frac{p G(d, \theta)}{\sigma^{2}}$ in (9a). Moreover, $\bar{g}(\bar{d})=\frac{p g(d)}{\sigma^{2}}$ in (9b). Consequently, if our dynamic EE-based 
allocation boundary occurs at $\bar{d}=D$, then according to (8), $D$ verifies $f(\bar{d}=D, \theta, \beta)=0$.

Proposition 1. The function $f(\bar{d}, \theta, \beta)$ in (8) is a monotonically increasing function of $\bar{d}$. Given that $\lim _{\bar{d}=0} f(\bar{d}, \theta, \beta)=-\infty$, it implies that, a boundary exists only if $\max _{\bar{d}, \theta, \beta} f(\bar{d}, \theta)>0$, or equivalently, $h(0.5, \beta) \leqslant\left(1+\max _{\theta} \bar{G}(0.5, \theta)\right)$, based on Fig. 2.

In other words, there exists $D>0$ such that $f(D, \theta, \beta)=0$; however, to ensure that the EE-based boundary is within the sector area, $D$ cannot be larger than half of the ISD. $D$ has to be a non-negative value to meet this condition. A detailed proof of Proposition 1 is provided in Appendix A.

According to (7), different power allocations can lead to different values of $\beta$, which corresponds to different boundaries. However, we can easily obtain the range of $\beta$ as follows. The first order derivative of (7) with respect to $p$ is

$$
\frac{\partial \beta}{\partial p}=\frac{\Delta(1-M) P_{c, m}}{\left(\Delta p+P_{c, m}\right)^{2}}
$$

therefore $\frac{\partial \beta}{\partial p}<0$, when $M>1$, is a decreasing function of $p$. Provided that $p$ is a non-negative value, it implies that $\beta$ has its maximum value when $p=0$, whereas $\beta$ has it minimum value when $p=+\infty$.

1) Maximum value of $\beta$ : $\beta_{\max }$ :

$$
\beta_{\max }=\lim _{p \rightarrow 0} \frac{\Delta p+M P_{c, m}}{\Delta p+P_{c, m}}=M .
$$

2) Minimum value of $\beta$ : $\beta_{\text {min }}$ : According to L'Hôpital's rule,

$\beta_{\text {min }}=\lim _{p \rightarrow \infty} \frac{\Delta_{m} p+M P_{c, m}}{\Delta_{m} p+P_{c, m}}=\lim _{p \rightarrow \infty} \frac{\partial\left(\Delta_{m} p+M P_{c, m}\right) / \partial p}{\partial\left(\Delta_{m} p+P_{c, m}\right) / \partial p}=1$

Given that the allocated power cannot exceed the pre-defined maximum transmit power $P^{\max }$, i.e. $p \in\left[0, P_{\max }\right]$, therefore, $\beta \in(1, M]$.

By calculating the first derivative of $f(\bar{d}, \theta, \beta)$ with respect to $\beta$, we can obtain that

$$
\frac{\partial f(\bar{d}, \theta, \beta)}{\partial \beta}=-\frac{\bar{g}(\bar{g}+1)^{\frac{1}{\beta}} \ln (\bar{g}+1)}{((\bar{g}+1)-1)^{2} \beta^{2}} .
$$

Provided that all the parameters in the above function are nonnegative, it implies that $f(\bar{d}, \theta, \beta)$ is a decreasing function of $\beta$, i.e.

$$
f\left(\bar{d}, \theta, \beta_{2}\right) \geqslant f\left(\bar{d}, \theta, \beta_{1}\right), \forall \beta_{1} \geqslant \beta_{2} .
$$

Given that $f(\bar{d}, \theta, \beta)$ is an increasing function of $\bar{d}$ (see Proposition 1) and that $f\left(\bar{d}, \theta, \beta_{2}\right) \geqslant f\left(\bar{d}, \theta, \beta_{1}\right)$, it implies that the inner area would be smaller when $\beta=\beta_{2}$ than when $\beta=\beta_{1}$ if the boundary exists in both cases.

Obtaining $D$ (the normalized distance at which the boundary occurs) requires to solve a polynomial equation with $\beta$ as an exponent, as it further explained in Section B of the Appendix. Hence, since $\beta$ falls in the range of $(1, M]$ which is not always an integer number, $D$ cannot be expressed in closed-form in the general case. However, in the case of $\beta=M$, a lower bound for the value of $D$ can be derived as follows.
Proposition 2. In a three-sector layout, let the boundary occurs at $\bar{d}=D$, then $D$ can be lower bounded as $D \geqslant$

$\underline{D}=\left(\frac{2 A}{\left(2+A 2^{n}\left(1+3^{-\frac{n}{2}}\right)\right)\left[-3+\sqrt{5+A 2^{n+2}\left(1+3^{-\frac{n}{2}}\right)}\right]^{+}}\right)^{\frac{1}{n}}$,

when $\beta=M$, and where $[x]^{+} \triangleq \max \{x, 0\}$.

The proof of Proposition 2 is given in Appendix B.

According to Proposition 2, $D$ is lower bounded by $\underline{D}$ when $\beta=M$. However, $\beta$ can be any number in the range of $(0, M]$. Given that $\beta$ becomes closer to $M$ as $p$ decreases in (11) and that green scheduling usually reduces $E_{b}$ by reducing $p$ [14], [37], $\underline{D}$ can be used as a starting point for finding the actual allocation boundary, $D$, as it is detailed in Corollary 1 .

Corollary 1. Provided that $f(\bar{d}, \theta, \beta)$ is an increasing function of $\bar{d}$, it then implies that

- Case 1: if $f(0, \theta, \beta)>0$ or $f(0.5, \theta, \beta)<0$, the boundary does not exist (i.e. there is no inner or outer area)

- Case 2: if $f(0, \theta, \beta) \leqslant 0$ and $f(\underline{D}, \theta, \beta) \leqslant 0$, the boundary exists, and $\underline{D} \leqslant D \leqslant 0.5, \forall \beta \in(0, M]$.

- Case 3: if $f(0, \theta, \beta) \leqslant 0$ and $f(\underline{D}, \theta, \beta) \geqslant 0$, the boundary exists, however $\underline{D} \geqslant D \geqslant 0, \forall \beta \in(0, M], \underline{D} \leqslant 0.5$.

Even though $D$ cannot be expressed in closed-form in the general case, based on Proposition 2, Corollary 1 and knowing, according to (11), that $\beta$ converges towards $M$ when the value of $p$ decreases, $D$ can be numerically obtained by performing a simple directional root search, where $\underline{D}$ is used as a starting value for the search.

\section{ENERGY-EFFICIENT BOUNDARY-ENABLED SCHEDULING FOR HETNET SYSTEMS}

In this section, we integrate the dynamic EE-based allocation boundary concept in the design of our scheduler as a means of improving both the EE and fairness in Hetnet systems. More specifically, we design a novel energy-efficient scheduler for minimizing (4), when considering a per-antenna transmit power constraint, by solving the following optimization problem

$$
\begin{array}{ll}
\min _{\mathbf{P}, \boldsymbol{\alpha}} & E_{b}(\mathbf{P}, \boldsymbol{\alpha}) \\
\text { s.t. } & \mathbf{P} \succeq 0, \\
& \sum_{s=1}^{S} p_{m}^{s}[t] \leq P_{m}^{\max }, \forall m \in \mathcal{M}=\{1, \ldots, M\} \text { and } \\
& t \in\{1, \ldots, T\} \\
& \alpha_{k(m)}^{s}[t] \in\{0,1\} .
\end{array}
$$

It can be remarked that (4) is not necessarily convex, even for $T=1$, because of the interference terms $I_{m, k(m)}^{s}[t]$ and, thus, solving (14) with (4) as an objective function is in general NP hard. However, by upper-bounding/approximating the objective function in (4), we can greatly simplify the problem in (14) and (4) and solve it in a low-complexity but sub-optimal manner, as it is further explained in the following. 


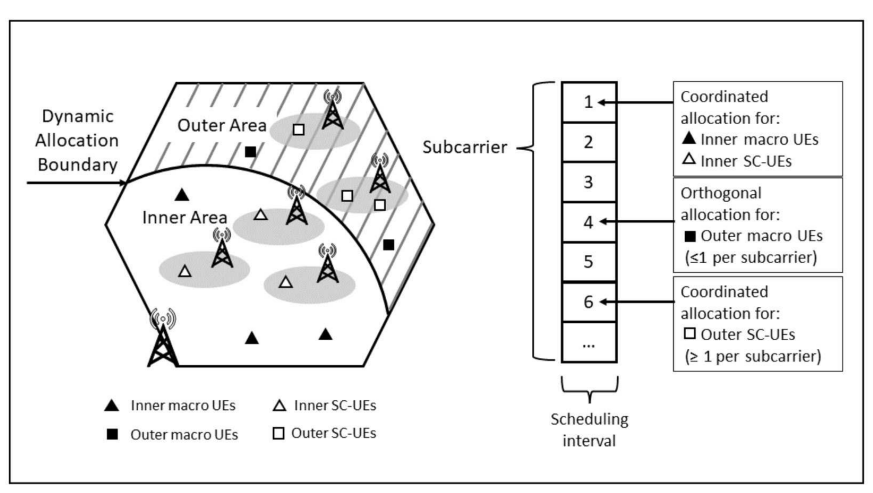

Fig. 3. Example of boundary-based scheduling

\section{A. Subcarrier/user Allocation}

In order to solve (14) a scheduler needs to acquire the CSI of all the links within a cluster during $T$ time intervals prior to perform the scheduling process. However, in practice, scheduling is normally performed on a time basis, i.e. when performing the scheduling at time instant $T$, the previous $T-1$ time instants have already been determined. Thus it is unlikely to optimize all the $T$ time instances of the scheduling decisions as in (4). Thus, instead of optimizing all the $T$ time instants of the scheduling decisions as in (14), we therefore optimize $\widetilde{E}_{b}(\mathbf{P}, \boldsymbol{\alpha})$ based on a time-slot-by-time-slot manner while taking into account the previous rate and power allocations as, for instance, in the PF scheduling scheme [21]:

$$
\widetilde{E}_{b}(\mathbf{P}, \boldsymbol{\alpha})=\frac{\sum_{v=1}^{t-1} P_{\Sigma}(\mathbf{P}[v])+P_{\mathrm{c}}[t]+\sum_{m=1}^{M} \Delta_{m} \sum_{s=1}^{S} p_{m}^{s}[t]}{\sum_{v=1}^{t-1} R_{\Sigma}(\mathbf{P}[v], \boldsymbol{\alpha}[v])+\sum_{m=1}^{M} \sum_{k=1}^{K} \mathcal{C}_{k(m)}[t]}
$$

instead of $E_{b}(\mathbf{P}, \boldsymbol{\alpha})$ in (14), at any time instance $t$. In effect, if all the past $T-1$ times scheduling decisions have been made, optimizing (15) is equivalent to optimizing (14).

The EE-based allocation boundary proposed in Section III dynamically partitioned (at each time instance $t$ ) the sector of a cell into two areas; the inner area where interference can be tolerated, and the outer area where interference should be avoided. In order to take this principle into account in our user/subcarrier allocation, we have designed a boundarybased user/subcarrier allocation strategy, which is illustrated in Fig. 3. In HetNet, the UEs are divided into macro or SC-UEs according to the cell they are associated with. In our scheme, these UEs are then further divided into inner or outer area UEs, resulting in four different types of UEs (see in Fig. 3) to be scheduled. According to our allocation boundary principle, inner UEs can be scheduled on the same subcarrier (coordinated allocation, e.g. subcarrier 1 in Fig. 3), where coordination is utilized to mitigate the interference. Whereas, in the outer area, each macro UE is scheduled on a dedicated subcarrier (orthogonal allocation, e.g. subcarrier 4 in Fig. 3) since better EE performance is achieved by avoiding interference from the neighbor cells. Meanwhile SC-UEs are scheduled with Macro UEs in the inner area (see subcarrier 1 in Fig. 3), i.e. underlay Hetnet, and amongst themselves (see subcarrier 6 in Fig. 3) in the outer area to avoid creating interference to the outer Macro
UEs.

Consequently, UEs can either be scheduled in an orthogonal or coordinated manner, where coordination allows to schedule more than one UE on the same subcarrier. Thus, in addition to the usual subcarrier and power allocation processes, a coordinated scheduler must group the users of different interfering cells in an effective manner for reducing interference. For instance in [14], the user grouping and subcarrier allocation are carried out simultaneously until the optimal grouping and allocation are found. The power allocation is carried out accordingly for every scheduling interval. However, this kind of scheduling is not efficient in terms of computational complexity especially when a large number of BSs are involved [38], such as in dense SC deployment. In order to reduce complexity, we follow a different approach where users of difference interfering cells are first grouped together and then assigned on a given subcarrier.

1) Inner area user grouping: In the inner area, macro and SCs users, which are scheduled on the same subcarrier by using coordinated scheduling (see Fig. 3), form a group that we refer as "inner area group". As pointed out in [15] for uniformly distributed users, the more users, the higher is the likelihood of having users with similar channel characteristics. We therefore create the group of users by selecting users from different cells with similar channel characteristics, i.e. having similar intended $g_{i, k(i)}^{s}$ and unintended, $g_{j, k(i)}^{s}$ channel gains, $j \neq i$. This is also in-line with the assumption we made in Section III for designing our scheduling boundary. However, due to the difference in characteristics of macro and SCs, we create two separate groups of users, i.e. macro and SC groups. In order to distinguish between the macro and SC parameters, we use the notations $\dot{\diamond}$ and $\ddot{\diamond}$, respectively, in the following. For simplicity of introduction, let us consider the case of three macro BSs in a cluster (as in Fig. 1); we aim at finding users $\mathrm{a}, \mathrm{b}$ and $\mathrm{c}$ in each sector denoted as 1, 2, 3 in Fig. 1, such that

$$
\begin{aligned}
& \dot{g}_{1, a(1)}^{s} \simeq \dot{g}_{2, b(2)}^{s} \simeq \dot{g}_{3, c(3)}^{s}, \\
& \dot{g}_{1, b(2)}^{s} \simeq \dot{g}_{2, c(3)}^{s} \simeq \dot{g}_{3, a(1)}^{s}, \\
& \dot{g}_{1, c(3)}^{s} \simeq \dot{g}_{2, a(1)}^{s} \simeq \dot{g}_{3, b(2)}^{s} .
\end{aligned}
$$

We apply the same user grouping principle to create a group of users for SCs. It then makes sense to allocate the same transmit power to the group of users having the same channel characteristics. Note that the macro BS usually transmits at a higher power than SC, such that macro and SC transmit antennas use different transmit powers. We therefore allocate the same transmit power $\dot{p}^{s}[t]$ for transmitting to the inner macro cell UEs, and the same transmit power $\ddot{p}^{s}[t]$ for transmitting to the inner SC-UEs. In turn, this simplifies the formulation of $\mathcal{C}_{k(m)}[t]$ in (1). Assuming that there are $M_{1}$ macro BSs and $M_{2}$ SCs in the inner area, $M_{1}+M_{2} \leqslant M$, the channel capacity of the $k$-th user served by BS $m$ on subcarrier $s$ for the macro inner tier is given by $\mathcal{C}_{k(m)}^{s}[t] \simeq \dot{\mathcal{C}}_{k(m)}^{s}[t]=$

$$
\alpha_{k(m)}^{s}[t] B \log _{2}\left(1+\frac{\dot{p}^{s}[t] \dot{g}_{m, k(m)}^{s}[t]}{\sigma^{2}+\dot{p}^{s}[t] \dot{G}_{m, k(m)}^{s}[t]+\ddot{p}^{s}[t] \dot{H}_{m, k(m)}^{s}[t]}\right),
$$


where $\dot{G}_{m, k(m)}^{s}[t]=\sum_{j=1, j \neq m}^{M_{1}} \dot{g}_{j, k(m)}^{s}[t]$ is the unintended channel gains from the other macro BSs, $\dot{H}_{m, k(m)}^{s}[t]=$ $\sum_{j=1}^{M_{2}} \dot{g}_{j, k(m)}^{s}[t]$ is the unintended channel gains from the SCs, which are also taken into account. Note that the notation $p_{j}^{s}[t]$ in (1) is such that $p_{j}^{s}[t]=\dot{p}^{s}[t]$ for any $j \in M_{1}$, and $p_{j}^{s}[t]=\ddot{p}^{s}[t]$ for any $j \in M_{2}$. Similarly, for the SC inner tier, $\mathcal{C}_{k(m)}^{s}[t]$ can be calculated by $\mathcal{C}_{k(m)}^{s}[t] \simeq \ddot{\mathcal{C}}_{k(m)}^{s}[t]=$

$$
\alpha_{k(m)}^{s}[t] B \log _{2}\left(1+\frac{\ddot{p}^{s}[t] \ddot{g}_{m, k(m)}^{s}[t]}{\sigma^{2}+\ddot{p}^{s}[t] \ddot{G}_{m, k(m)}^{s}[t]+\dot{p}^{s}[t] \ddot{H}_{m, k(m)}^{s}[t]}\right),
$$

where $\ddot{G}_{m, k(m)}^{s}[t]=\sum_{j=1, j \neq m}^{M_{2}} \ddot{g}_{j, k(m)}^{s}[t]$ is the unintended channel gains from the other SCs, $\ddot{H}_{m, k(m)}^{s}[t]=$ $\sum_{j=1}^{M_{1}} \ddot{g}_{j, k(m)}^{s}[t]$ is the unintended from the macro BSs.

In addition, given that the channel characteristics of users in the same group are not always fully similar, instead of using $g_{m, k(m)}^{s}[t], G_{m, k(m)}^{s}[t]$ and $H_{m, k(m)}^{s}[t],(16)$ and (17) can be further simplified by using the aggregate intended and unintended channel gains which are defined as the average intended and unintended channel gains of the same group of users, we respectively define them as

$$
\begin{aligned}
& \dot{g}_{k}^{s}[t]=\frac{1}{M_{1}} \sum_{m=1}^{M_{1}} \dot{g}_{m, k(m)}^{s}[t], \quad \ddot{g}_{k}^{s}[t]=\frac{1}{M_{2}} \sum_{m=1}^{M_{2}} \ddot{g}_{m, k(m)}^{s}[t], \\
& \dot{G}_{k}^{s}[t]=\frac{1}{M_{1}} \sum_{m=1}^{M_{1}} \dot{G}_{m, k(m)}^{s}[t], \quad \ddot{G}_{k}^{s}[t]=\frac{1}{M_{2}} \sum_{m=1}^{M_{2}} \ddot{G}_{m, k(m)}^{s}[t], \\
& \dot{H}_{k}^{s}[t]=\frac{1}{M_{2}} \sum_{m=1}^{M_{2}} \dot{H}_{m, k(m)}^{s}[t], \quad \ddot{H}_{k}^{s}[t]=\frac{1}{M_{1}} \sum_{m=1}^{M_{1}} \ddot{H}_{m, k(m)}^{s}[t] .
\end{aligned}
$$

2) Outer area user grouping: In the outer area, we distinguish between the macro and SC-UEs.

(i) Outer macro users are scheduled based on the orthogonal allocation principle, such that at most one outer macro UE is scheduled on a subcarrier (see Fig. 3); thus, the channel capacity of each outer macro UEs can be expressed as in (16), but where $\dot{G}_{m, k(m)}^{s}[t]=0$ and $\dot{H}_{m, k(m)}^{s}[t]=0$. Indeed, given that each outer macro UE is scheduled on its own on a subcarrier, it does not suffer from any interference.

(ii) As far as outer SC-UEs are concerned, their channel capacity can be expressed as in (17), but where $\ddot{H}_{m, k(m)}^{s}[t]=0$, since that they are not interfering with outer macro UEs.

\section{B. Power Allocation}

Having first grouped the UEs and assigned a group of UEs (or a user) on each subcarrier, minimizing $E_{b}$ in (14) then requires to find an energy efficient power allocation. The simplification of (1) into (16) and (17) derived from our user grouping is not only useful for subcarrier allocation, but it is even more advantageous for power allocation. Indeed, by inserting (16) and (17) combining with the aggregate gains into $(15), \widetilde{E}_{b}(\mathbf{P}, \boldsymbol{\alpha})$ can be re-expressed as $\widetilde{E}_{b}(\mathbf{P}, \boldsymbol{\alpha})=$

$$
\frac{P_{0}[t]+\sum_{m=1}^{M_{1}} \dot{\Delta}_{m} \sum_{s=1}^{S} \dot{p}^{s}[t]+\sum_{m=1}^{M_{2}} \ddot{\Delta}_{m} \sum_{s=1}^{S} \ddot{p}^{s}[t]}{R_{0}[t]+\sum_{m=1}^{M_{1}} \sum_{s=1}^{S} \dot{\mathcal{C}}_{s, k(m)}[t]+\sum_{m=1}^{M_{2}} \sum_{s=1}^{S} \ddot{\mathcal{C}}_{s, k(m)}[t]},
$$

where $P_{0}[t]=\sum_{v=1}^{t-1} P_{\Sigma}(\mathbf{P}[v])+\sum_{m=1}^{M} \sum_{s=1}^{S} P_{c, m}^{s}[t]$ as well as $R_{0}[t]=\sum_{v=1}^{t-1} R_{\Sigma}(\mathbf{P}[v], \boldsymbol{\alpha}[v])$ are fixed terms, which turns out to be a pseudo-convex function if one of the powers (either $\dot{p}^{s}[t]$ or $\ddot{p}^{s}[t]$ ) is fixed. Consequently, given a fixed user scheduling decision $\boldsymbol{\alpha}$, obtaining an energy-efficient power allocation for a particular tier can be done in a low-complexity manner.

Proposition 3. For a fixed $\boldsymbol{\alpha}$, let $\mathbf{P}$ be a stationary point of $\widetilde{E}_{b}(\mathbf{P}, \boldsymbol{\alpha})$ in (19), i.e. $\nabla \widetilde{E}_{b}(\mathbf{P}, \boldsymbol{\alpha})=\mathbf{0}$, then according to (19), the optimal unconstrained value of $p^{s}[t]$ for the macro tier, $\dot{p}^{s}[t]$ can be obtained by solving the following quartic function as

$$
\left(\dot{p}^{s}[t]\right)^{4}+A\left(\dot{p}^{s}[t]\right)^{3}+B\left(\dot{p}^{s}[t]\right)^{2}+C\left(\dot{p}^{s}[t]\right)+D=0,
$$

where the coefficients $A, B, C, D$ can be obtained by rearranging (22). The quartic function for solving $\ddot{p}^{s}[t]$ for the SCs can be formulated in the same way.

Proof: From (19),

$$
\begin{aligned}
& \frac{\partial \widetilde{E}_{b}(\mathbf{P}, \boldsymbol{\alpha})}{\partial \dot{p}^{s}[t]}=\frac{\widetilde{E}_{b}(\mathbf{P}, \boldsymbol{\alpha})}{P_{0}[t]+\sum_{m=1}^{M_{1}} \dot{\Delta}_{m} \sum_{s=1}^{S} \dot{p}^{s}[t]+\sum_{m=1}^{M_{2}} \ddot{\Delta}_{m} \sum_{s=1}^{S} \ddot{p}^{s}[t]} \\
& \times\left[\Delta_{m}-\widetilde{E}_{b}(\mathbf{P}, \boldsymbol{\alpha}) \frac{B}{\ln (2)}\left(\frac{\dot{G}_{k}^{s}[t]+\dot{g}_{k}^{s}[t]}{\sigma^{2}+\dot{p}^{s}[t] \dot{G}_{k}^{s}[t]+\ddot{p}^{s} \dot{H}_{k}^{s}+\dot{p}^{s}[t] \dot{g}_{k}^{s}[t]}\right.\right. \\
& -\frac{\ddot{H}_{k}^{s}[t]}{\sigma^{2}+\dot{p}^{s}[t] \dot{G}_{k}^{s}[t]+\ddot{p}^{s} \dot{H}_{k}^{s}}+\frac{\ddot{H}_{k}^{s}[t]}{\sigma^{2}+\dot{p}^{s}[t] \ddot{H}_{k}^{s}[t]+\ddot{p}^{s} \ddot{G}_{k}^{s}+\ddot{p}^{s}[t] \ddot{g}_{k}^{s}[t]} \\
& \left.\left.-\frac{\dot{p}^{2}+\dot{p}^{s}[t] \ddot{H}_{k}^{s}[t]+\ddot{p}^{s} \ddot{G}_{k}^{s}}{\sigma^{s}}\right)\right],
\end{aligned}
$$

when $\alpha_{\dot{k}(m)}^{s}[t]=1$, such that $\frac{\partial \widetilde{E}_{b}(\mathbf{P}, \boldsymbol{\alpha})}{\partial \dot{p}^{s}[t]}=0$ is equivalent to

$$
\frac{1}{\widetilde{E}_{b}(\mathbf{P}, \boldsymbol{\alpha})}=\frac{B}{\ln (2) \dot{\Delta}_{m}}\left(C_{1}-C_{2}+C_{3}-C_{4}\right),
$$

where

$$
\begin{gathered}
C_{1}=\left(\frac{\sigma^{2}+\ddot{p}^{s}[t] \dot{H}_{k}^{s}[t]}{\dot{G}_{k}^{s}[t]+\dot{g}_{k}^{s}[t]}+\dot{p}^{s}[t]\right)^{-1}, C_{2}=\left(\frac{\sigma^{2}+\ddot{p}^{s}[t] \dot{H}_{k}^{s}[t]}{\dot{G}_{k}^{s}[t]}+\dot{p}^{s}[t]\right)^{-1}, \\
C_{3}=\left(\frac{\sigma^{2}+\ddot{p}^{n}[t] \dot{H}_{k}^{n}[t]+\ddot{p}_{k}^{n}[t] \ddot{g}_{k}^{n}[t]}{\ddot{H}_{k}^{n}[t]}+\dot{p}^{n}[t]\right)^{-1}, \\
C_{4}=\left(\frac{\sigma^{2}+\ddot{p}_{k}^{n}[t] \ddot{g}_{k}^{n}[t]}{\ddot{H}_{k}^{n}[t]}+\dot{p}^{n}[t]\right)^{-1},
\end{gathered}
$$

(20) is then obtained by rearranging the latter.

In addition, the power allocation for the users in the outer area can be obtained by following the same principle as above. Since there is no inter-tier interference, the optimal power allocation for the outer macro UEs and outer SC-UEs can be easily obtained respectively by

$$
\dot{p}^{s}[t]=\frac{\mu}{\dot{\Delta}_{m} \ln (2)}-\frac{1}{\dot{g}_{m, k(m)}^{s}[t]},
$$


and $\ddot{p}^{s}[t]=\frac{\sigma^{2}}{2} \times$

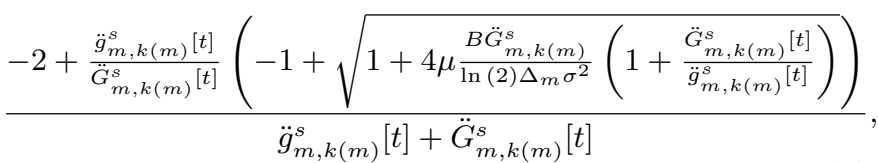

where $\mu=\widetilde{E}_{b}(\mathbf{P}, \boldsymbol{\alpha})$.

\section{Scheduler Algorithm}

Our proposed boundary-enabled scheduling is composed of four stages, as it is illustrated in Fig. 4.

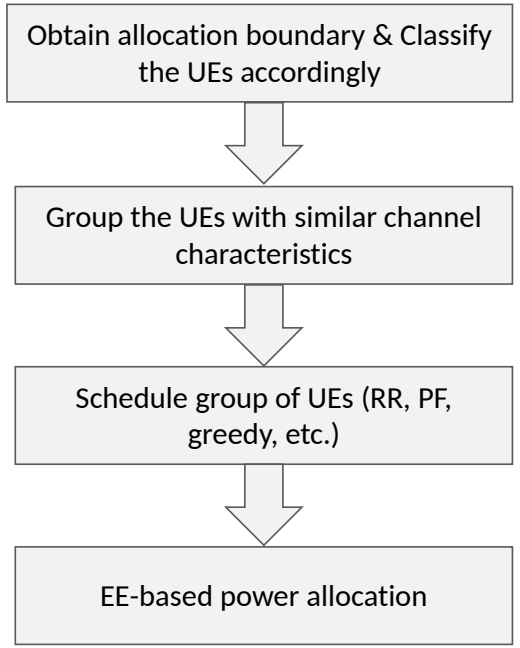

Fig. 4. Block diagram of boundary-enabled scheduling.

1) Dynamic Allocation Boundary and UE Classification: The first stage of the boundary-enabled scheduling process computes the dynamic EE-based allocation boundary. For a given network layout, the value of $\underline{D}$ in Proposition 2 is first calculated; it is then used to search for the actual allocation boundary $D$. This allocation boundary served as a classifying criterion to differentiate between the UEs, i.e. inner or outer UEs. In addition, UEs are further classified as macro and SC-UEs according to the cell they are associated with.

2) User Grouping: After classifying the UEs, we apply our user grouping principle to group the UEs, i.e. UEs with similar channel characteristics will be grouped together to form a scheduling group. For the inner area UEs, macro and SCUEs are scheduled on the same subcarrier, however due to the difference in both the users' channel characteristics and BS type, macro UE groups and SC-UE groups are created separately.

3) Subcarrier Assignment: Once the UE groups are formed, it then needs to be decided which group of UEs shall be assigned for each subcarrier. In this stage, existing scheduling schemes such as greedy, PF, or RR can be used, however, with modified selection criteria to accommodate the different categories of UEs, as it is further detailed below.

(i) Greedy scheduling assigns to a subcarrier $s$ the user/group of users providing the best average achievable user rate on
1: function EEPA-LC( $\left(M_{1}, M_{2}, S, \mathbf{g}[t], \boldsymbol{k}[t], P_{0}[t], R_{0}[t], W, \sigma^{2}, \Delta_{m}, \mathbf{P}_{m}^{\max }\right)$ Set $\mu$ as in (19), $p_{m}^{s}=P_{m}^{\max } / S$;

Set $x=\mu+1$ and $\epsilon=10^{-6}$;

while $W|\mu-x|>\epsilon$ do

Obtain $\dot{p}^{s}[t]$ for inner area macro UEs by solving the quartic function (20), $\forall s \in \mathcal{S}, \dot{g}^{s}[t], \dot{G}^{s}[t]$ and $\dot{H}^{s}[t]$ can be obtained by (18);

6: $\quad$ Obtain $\ddot{p}^{s}[t]$ for inner area SC-UEs by following the same principle as as in (20), $\forall s \in \mathcal{S}, \ddot{g}^{s}[t], \ddot{G}^{s}[t]$ and $\ddot{H}^{s}[t]$ can be obtained by (18);

7: $\quad$ For outer area macro and SC-UEs, obtain $\dot{p}^{s}[t]$ and $\ddot{p}^{s}[t]$ by (23) and (24), respectively;

8: $\quad$ Set $x=\mu$;

9: $\quad$ Compute $\mu=\widetilde{E}_{b}(\mathbf{P}, \boldsymbol{\alpha})$ by inserting $\dot{p}^{s}[t]$ and $\ddot{p}^{s}[t]$ into (15), $\dot{g}^{s}[t], \dot{G}^{s}[t], \dot{H}^{s}[t], \ddot{g}^{s}[t], \dot{G}^{s}[t]$ and $\dot{H}^{s}[t]$ are obtained by (18) in (15);

end while

for $m=1: M$ do Set $p_{m}^{s}[t]=\frac{G^{s}[t]+H^{s}[t]}{\sum_{j=1, j \neq m}^{M} g_{m, k^{s}[t](j)}^{s}[t]}, \forall s \in \mathcal{S}$;

end for

Set $\Sigma^{s}=\sum_{m=1}^{M} p_{m}^{s}[t], \forall s \in \mathcal{S}$;

for $m=1: M$ do Set $p_{m}^{s}[t]=\frac{M p^{s}[t] p_{m}^{s}[t]}{\Sigma^{s}}, \forall s \in \mathcal{S}$; if $\sum_{s=1}^{S} p_{m}^{s}[t]>P_{m}^{\max }$ then $\quad \triangleright$ Power Constraint end if Set $p_{m}^{s}[t]=\frac{p_{m}^{s}[t] P_{m}^{\max }}{\sum_{s=1}^{S} p_{m}^{s}[t]}, \forall s \in \mathcal{S}$;

end for

return $p_{m}^{s}[t]$, for any $s \in \mathcal{S}$ and $m \in \mathcal{M}$; end function

this particular subcarrier, such that based on (16) and (17)

$$
k^{s}(m)=\arg \max _{k(m) \in K(m)} \overline{\mathcal{C}}[t],
$$

where $\overline{\mathcal{C}}^{s}[t]=\frac{\sum_{m=1}^{M_{1}} \dot{\mathcal{C}}_{k(m)}^{s}[t]+\sum_{m=1}^{M_{2}} \ddot{\mathcal{C}}_{k(m)}^{s}[t]}{M_{1}+M_{2}}$ in the inner area, i.e. the achievable rate of the scheduled groups divided by the number of BSs. The same principle applies to the outer area UEs.

(ii) Proportional fair scheduling relies on the PF criterion [21], which is defined as

$$
k^{s}(m)=\arg \max _{k(m) \in K(m)} \frac{\overline{\mathcal{C}}^{s}[t]}{\mathcal{T}^{s}},
$$

where $\mathcal{T}^{s}=\sum_{t=1}^{T-1} \overline{\mathcal{C}}^{s}[t], \mathcal{T}^{s}$ is the sum of the previous average achievable rate of the scheduled user groups.

(iii) Round robin scheduling [20], once the user groups are formed, allocates sequentially the subcarriers to each group of UEs until no subcarriers are available; each user group therefore receives a similar number of subcarriers.

4) Low-complexity EE-based Power Allocation: The last stage of the scheduling process is to allocate power to the scheduled groups of UEs. Given that $\widetilde{E}_{b}(\mathbf{P}, \boldsymbol{\alpha})$ in (19) is pseudo-convex if one of the powers ( $\dot{p}^{s}[t]$ or $\left.\ddot{p}^{s}[t]\right)$ is fixed, $\dot{p}^{s}[t]$ and $\ddot{p}^{s}[t]$ can be expressed as two quartic functions of a single variable $\mu$, respectively. The low-complexity power allocation algorithm is described in the "EEPA-LC" function for obtaining $\dot{p}^{s}[t]$ and $\ddot{p}^{s}[t]$. Starting from equal power allocation, i.e. allocating the same power $P_{m}^{\max } / S$ on each subcarrier (where $P_{m}^{\max }=\dot{P}^{\max }$ and $\ddot{P}^{\max }$ for macro and SCs respectively), then a simple iterative process (as seen in [39]) is performed for obtaining $\dot{p}^{s}[t]$ and $\ddot{p}^{s}[t]$. At this point, $\dot{p}^{s}[t]$ and $\ddot{p}^{s}[t]$ are 
computed as if the channel gains of the users selected on subcarrier $s$ are symmetric. However, since it is not generally the case, we need to refine the per-subcarrier transmit power of each BS by using the aggregate channel gains to compute the power allocation and obtain $\widetilde{E}_{b}$. In addition, we use a simple normalization as $p_{m}^{s}=\frac{p_{m}^{s} P_{m}^{\max }}{\sum_{s=1}^{S} p_{m}^{s}}$ (at line 18 of the "EEPA-LC" function) to ensure that the total transmit power of each BS does not violate the power constraint in (14). Note that $\mathrm{g}[t]$ (one of the inputs of "EEPA-LC") is a vector that contains all the $g_{m, k(m)}^{s}[t]$ channel gain, $\forall m \in \mathcal{M}, s \in \mathcal{S}=\{1, \ldots, S\}$, $M_{1}$ and $M_{2}$ represents the number of macro and SCs, $k(m)$ represents the UE index of BS $m$ being scheduled on a given subcarrier, and $\mathbf{P}_{m}^{\max }$ is a vector which contains the maximum power constraint for each BS.

\section{Complexity}

Generally, green scheduling schemes include both subcarrier and power allocation. The complexity of green scheduling schemes is related to three parameters, the number of users (i.e. $K$ ) to be scheduled, the number of BSs (i.e. $M$ ) and the number of subcarriers (i.e. $S$ ). For subcarrier allocation, the complexity depends on the user scheduling scheme being used. For instance, when greedy scheduler is used, the complexity of finding the user among $K$ users having the highest EE performance is $\mathcal{O}(K)$. While the complexity of the RR scheduler is $\mathcal{O}(1)$. In terms of power allocation, the power allocation is updated for each BS, the complexity grows linearly with the number of BSs, i.e. $\mathcal{O}(M)$ for iterative-based algorithm (such as Algorithm 5 in [14]). Whereas in our algorithm, the power allocation is relevant to the number of tiers (i.e. macro and small tiers) rather than the number of BSs, which is $\mathcal{O}(1)$. Finally, in terms of the number of subcarriers, the complexity is $\mathcal{O}(S)$, since for each subcarrier, a power allocation needs to be determined.

\section{Numerical Results}

In order to showcase the benefits of our novel energyefficient boundary-enabled scheduling scheme for HetNet in terms of EE and user fairness, we compare its performance, in terms of relevant metrics, against existing green scheduling methods [14], [38] in a HetNet setting with either underlay or overlay SC deployment. Our results, which have been obtained through Monte-Carlo simulations, depict the global energy per bit, the average per-sector transmit power and rate as well as the average per UE fairness for $T=1000$ time instances.

\section{A. Simulation Parameters and Settings}

We consider a realistic downlink setting where the channel gain, $g_{i, k(m)}^{s}$, is composed of three components, namely, the Rayleigh fading, pathloss and shadowing. Regarding the latter, we use the 3GPP pathloss and shadowing model, as reported in Table 27 and Table 32 of [40] for macro and SCs, respectively, where the pathloss + shadowing coefficients between the $i$-th BS and the $k$-th user of sector $m$ is given by

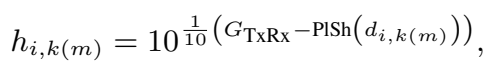

with $G_{\mathrm{TxRx}}$ being the antenna gain of the BS-UE transmission. In addition, $d_{i, k(m)}$ represents the distance between BS $i$ and the $k$-th user of sector $m$ and $\operatorname{PlSh}\left(d_{i, k(m)}\right)=\mathrm{P}_{\mathrm{LOS}}\left(d_{i, k(m)}\right) \mathrm{PL}_{\mathrm{LOS}}\left(d_{i, k(m)}\right)+(1-$ $\left.\mathrm{P}_{\mathrm{LOS}}\left(d_{i, k(m)}\right)\right) \mathrm{PL}_{\mathrm{NLOS}}\left(d_{i, k(m)}\right)$ is the path-loss + shadowing function. Moreover, $\mathrm{P}_{\mathrm{LOS}}$ is the line-of-sight (LOS) probability, and $\mathrm{PL}_{\mathrm{LOS}}$ and $\mathrm{PL}_{\mathrm{NLOS}}$ are the LOS and non-LOS (NLOS) distance dependent path-loss + shadowing functions, whose expressions can be found in Table 27 and Table 32 of [40] for the urban macro and SC scenarios.

In order to simulate the system and compute the pathloss and shadowing coefficients, we set the carrier frequency $f_{c}=$ $2.1 \mathrm{GHz}$, the number of subcarriers $S=600$ (by default) shared by 20 uniformly distributed UEs per sector, where each subcarrier has a $B=15 \mathrm{kHz}$ bandwidth, the noise spectral density $N_{0}=-165.2 \mathrm{dBm} / \mathrm{Hz}$, the antenna gain $G_{\mathrm{TxRx}}=$ $14 \mathrm{dBi}$ for macro BSs and $5 \mathrm{dBi}$ for small BSs, the macro BS height $h_{\mathrm{BS}}=35 \mathrm{~m}$ and the small $\mathrm{BS}$ height is $10 \mathrm{~m}$, the average building height $h_{\mathrm{av}}=5 \mathrm{~m}$, the street width $W_{\mathrm{St}}=20 \mathrm{~m}$, and the UE height $h_{\mathrm{UT}}=1.5 \mathrm{~m}$. Note that $\sigma^{2}=N_{0} B$.

Concerning the power model parameters, according to Table 2 of [4], we set $P_{\mathrm{BS}}^{C i}=130 \mathrm{~W}, \Delta_{m}=4.7$ and $P_{m}^{\max }=20 \mathrm{~W}, \forall m \in \mathcal{M}_{1}$ for the macro BS, whereas $P_{\mathrm{BS}}^{C i}=6.8 \mathrm{~W}, \Delta_{m}=4$ and $P_{m}^{\max }=0.13 \mathrm{~W}, \forall m \in \mathcal{M}_{2}$ for the small BS. In addition, we set $P_{\mathrm{UE}}^{C i}=100 \mathrm{~mW}$ according to [41]. Furthermore, we consider that SCs can enter sleep mode when no UEs are within its serving area, thus the consumed power in sleep mode are neglected. Note that as in [14], [15], the extra energy consumption due to the coordination process has been considered negligible.

\section{B. Simulation Results and Insights}

In order to illustrate the reliability and low-complexity of our energy-efficient scheduling scheme denoted as "Bound-EEPALC", its performance is compared, in a two-tier HetNet system (see in Fig. 1), against three relevant schemes, i.e. the "CoordEEPA-Ven", "FFR-EEPA-LC" as well as "Overlay-EEPA-Ven" schemes, as a function of ISD. The "Coord-EEPA-Ven" is an energy-efficient coordinated scheduling scheme that has been developed in [14], more precisely in Algorithm 5 of [14]. Whereas the "FFR-EEPA-LC" scheme adopts the FFR used in [25] along with "EEPA-LC" power allocation instead of full power allocation. Moreover, "Overlay-EEPA-Ven" splits the whole frequency band into two halves as for instance in [16][18], where macro and SCs are operating on separate subbands such that cross-tier interferences are avoided; each tier relies on the power allocation method from [14], which we denote as "EEPA-Ven". The comparison of these schemes is undertaken for a total number of $15 \mathrm{SCs}$ deployed in the three-sector layout of Fig. 1, and when utilizing either greedy, PF or RR for performing subcarrier assignment.

We compare the "Bound-EEPA-LC", "FFR-EEPA-LC", "Coord-EEPA-Ven" and "Overlay-EEPA-Ven" schemes in terms of the average transmit power and for various user/subcarrier allocation methods in Fig. 5. In general, these four schemes with energy-efficient power allocation have a relative low transmit power for each sector (less than $1.5 \mathrm{~W}$ ). It can been seen that 


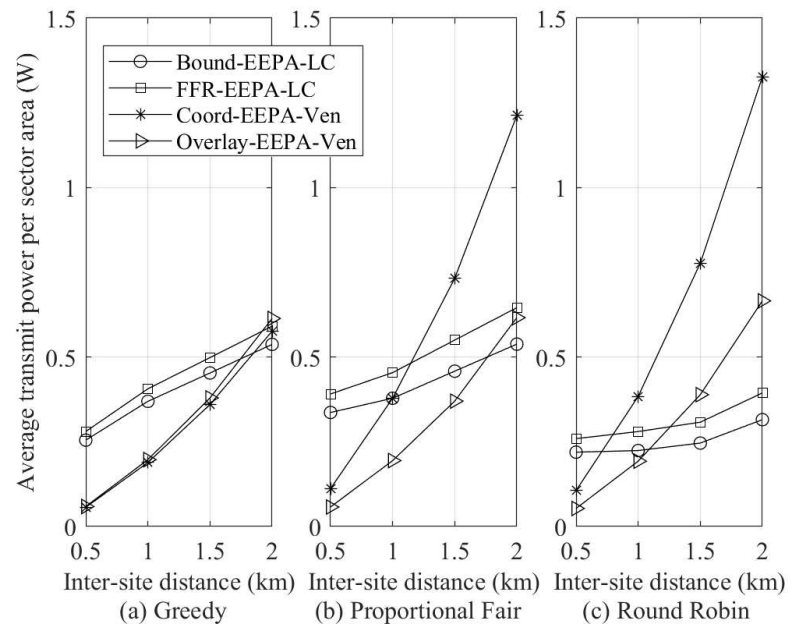

Fig. 5. Average transmit power per sector performance in the downlink of HetNet.

as the ISD increases, the transmit powers of all the schemes increase. Since macro UEs are further to their serving BSs when ISD is larger, a higher transmit power is needed to serve these users. It can also be observed in the figure that, as ISD increases, the transmit powers of "Coord-EEPA-Ven" and "Overlay-EEPA-LC" schemes increase at a faster rate than "Bound-EEPA-LC" and "FFR-EEPA-LC" schemes. This is because "EEPA-Ven" does not take into consideration the different power characteristics of the macro and small BSs, the power allocation for the macro UEs is driven by the SC-UEs when ISD is small (i.e. high interference), whereas it is less affected when ISD is larger (i.e. low interference).
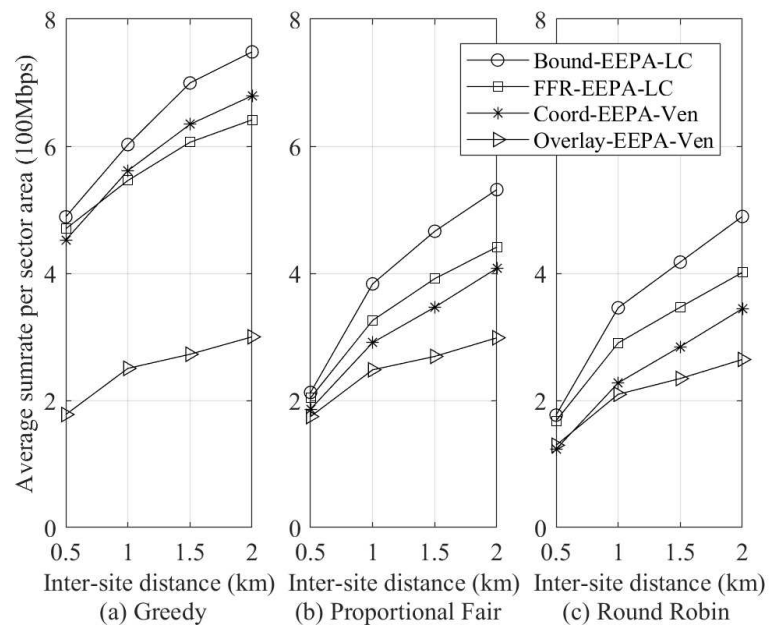

Fig. 6. Average sum rate per sector performance in the downlink of HetNet.

Figure 6 illustrates the average sum rate performance of the four schemes. It can be observed that our "Bound-EEPA-LC" scheme exhibits the best overall rate performance regardless of the subcarrier assignment. As it can be observed in Fig. 6 (a), when greedy allocation is being used, "Coord-EEPA-Ven" performs better than "FFR-EEPA-LC" when ISD is large. This is because when greedy allocation is used, users with good channel conditions are usually being scheduled, since these users are more robust to interferences, it is unnecessary to avoid interferences as in "FFR-EEPA-LC". However, when the interference is strong (i.e. ISD is small), or the users with poor channel conditions are also being scheduled (i.e. PF or $\mathrm{RR}$ ), interference avoidance is a better choice. As it can be seen in Figs. 6 (b) and (c), "FFR-EEPA-LC" outperforms "Coord-EEPA-Ven". Amongst the four schemes using energyefficient power allocation, "Overlay-EEPA-Ven" has the worst rate performance due to the lower utilization of the spectrum resources (i.e. each tier is only allowed to utilize half of the spectrum resources compared to "Coord-EEPA-Ven"). However, as it can be seen in Fig. 6 (c), "Overlay-EEPAVen" outperforms "Coord-EEPA-Ven" when interference is high (i.e. ISD $=0.5 \mathrm{~km}$ ). It can also be observed in all the sub-figures that the rate increases as the ISD increases due to the reduced interference.

Figure 7 shows the average energy per bit power consumption performance. It can be observed that "Bound-EEPA-LC" achieves the best overall $E_{b}$ performance. When greedy allocation is being used, "Bound-EEPA-LC", "Coord-EEPA-Ven" and "FFR-EEPA-LC" have similar EE performance. Although "Bound-EEPA-LC" and "FFR-EEPA-LC" have similar transmit power when using greedy and PF (see in Figs. 5 (a) and (b)), better rate performance can be achieved by scheduling more users with good channel conditions (i.e. greedy), which results in a better EE performance. In addition, it can be observed from all the subfigures, "Bound-EEPA-LC" outperforms "FFR-EEPALC" regardless of the user/subcarrier allocation methods that are being used, this implies that dynamic allocation of the resources can further enhance the EE performance in comparison with fixed allocation. Comparing to "Overlay-EEPA-Ven", up to $63 \%$ performance gain can be achieved by "Bound-EEPA-LC".

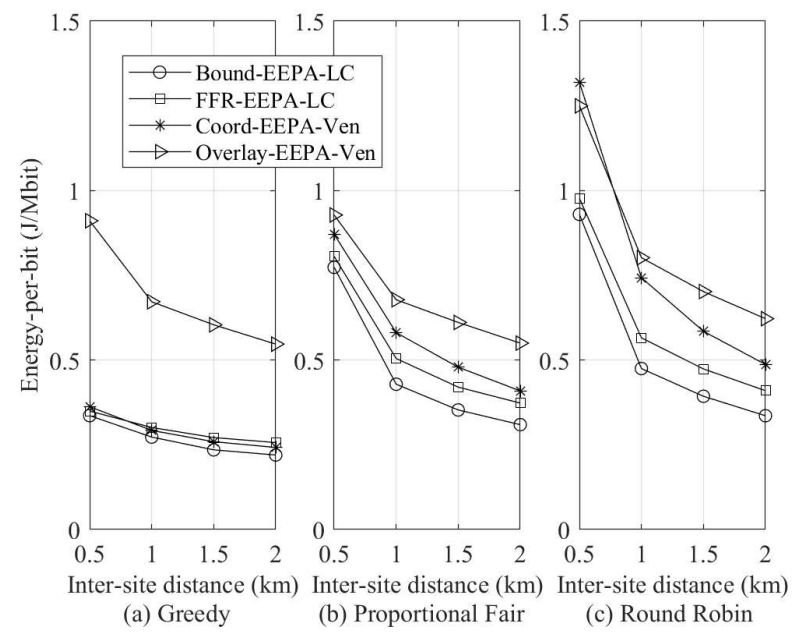

Fig. 7. Average $E_{b}$ per sector performance in the downlink of HetNet.

Figure 8 shows the fairness performance of the four schemes, where the Jain's fairness index is used to calculate the fairness of rate distribution among the UEs. It can be seen in Fig. 8 that "Bound-EEPA-LC" can achieve the best user fairness amongst all the schemes, whereas "Coord-EEPA-Ven" performs the worst. In "Bound-EEPA-LC" and "FFR-EEPA-LC", outer 
area macro UEs are protected from intra-tier and inter-tier interferences; whereas in "Overlay-EEPA-Ven" macro and SCUEs are protected from inter-tier interference since they are scheduled on orthogonal subbands. However, in "Coord-EEPAVen", outer area macro UEs are suffering from both intra and inter-tier interferences, resulting in poor user fairness. When observing the three subfigures, it can be seen that the fairness performances using different user/subcarrier allocation methods are similar. These results indicate that, different from the traditional single tier network where fairness is only related to the criterion being used to select the users (e.g. maximum SINR (greedy)), user fairness in HetNet is also related to the type of cell (i.e. macro or SC) used to serve the users. For instance, UEs within the same SC are likely to have similar channel conditions (i.e. similar rates) due to the closer distance between SC-UEs and the SC. SC-UEs therefore are less sensitive to the user/subcarrier allocation methods (i.e. greedy, PF, RR) being used as compared to macro UEs, where channel conditions of macro UEs within the same cell can vary quite differently.

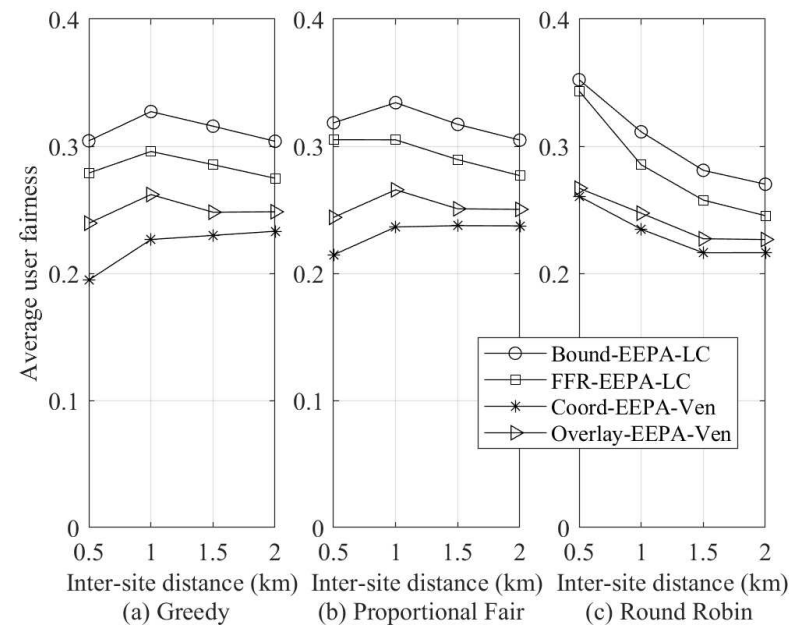

Fig. 8. Average user fairness performance in the downlink of HetNet.

\section{CONCLUSION}

In this paper, we proposed a novel green scheduling framework to improve both the EE and user fairness of underlay heterogeneous networks. We propose a new concept named "scheduling boundary" to dynamically categorize the users into inner and outer area users and improve the overall user fairness. This scheduling boundary has then been integrated into the design of our scheduler. A bespoke energy-efficient power allocation algorithm is also provided, which coordinates the transmit powers for both macro and small BSs, to jointly improve the EE and user fairness. Our boundary-enabled scheduling algorithm is then compared against existing green scheduling schemes, and the numerical results demonstrated that our proposed boundary-enabled scheduling scheme can reduce the energy per-bit consumption by up to $63 \%$ while still achieving better user fairness. Moreover, our scheduling algorithm exhibits low complexity that is suitable to be applied in densely deployed SCs. Furthermore, with the development of new techniques such as non-orthogonal multiple access scheme, where more users can be served at the same time on the same subcarrier with appropriate scheduling. Further investigations are needed to identify the benefits brought by these new technologies to improve the EE and well as user fairness in communication networks.

\section{ACKNOWLEDGMENT}

The authors would also like to acknowledge the support of the University of Surrey's 5G Innovation Centre (5GIC) (http://www.surrey.ac.uk/5gic) members for this work.

\section{APPENDIX A \\ PROOF OF PROPOSITION 1}

Proving the existence of the dynamic allocation boundary is equivalent to proving that the gain function $f(\bar{d}, \theta)$ is a monotonically increasing function of $\bar{d}$.

According to (8), the first order derivative of $G(\bar{d}, \theta)$ can be expressed as

$$
\frac{\partial \bar{G}(\bar{d}, \theta)}{\partial \bar{d}}=A n\left(\frac{a-\bar{d}}{\left[(\bar{d}-a)^{2}+b^{2}\right]^{\frac{n}{2}+1}}+\frac{c-\bar{d}}{\left[(\bar{d}-c)^{2}+e^{2}\right]^{\frac{n}{2}+1}}\right) .
$$

Note that when $\theta$ is in the interval $\left[0, \frac{\pi}{3}\right]$, we have $a, c \in$ $[0.5,1]$, such that $a$ and $c$ are always larger than or equal to the maximum value of $\bar{d}$, i.e. 0.5 . Since $A$ and $n$ are non-negative, it implies that

$$
\frac{\partial \bar{G}(\bar{d}, \theta)}{\partial \bar{d}} \geqslant 0, \forall \theta \in\left[0, \frac{\pi}{3}\right], \forall \bar{d} \in(0,0.5] .
$$

Meanwhile, $\frac{\partial \bar{G}(\bar{d}, \theta)}{\partial d}<0$ when $\theta \in\left(\frac{\pi}{3}+\frac{\pi}{4}, \frac{2 \pi}{3}\right]$. According to (9b), the first order derivative of $h(\bar{d}, \beta)$ can be calculated as

$$
\frac{\partial h(\bar{d})}{\partial \bar{d}}=\frac{\frac{\partial \bar{g}}{\partial d}\left[(1+\bar{g})^{\frac{1}{\beta}}-1\right]-\bar{g} \frac{1}{\beta} \frac{\partial \bar{g}}{\partial d}(1+\bar{g})^{\frac{1}{\beta}-1}}{\left[(1+\bar{g})^{\frac{1}{\beta}}-1\right]^{2}} .
$$

Given that

$$
\frac{\partial \bar{g}(\bar{d})}{\partial \bar{d}}=-n A \bar{d}^{-(n+1)}=-n \bar{d}^{-1} \bar{g},
$$

we then get

$$
\frac{\partial h(\bar{d}, \beta)}{\partial \bar{d}}=\frac{-n \bar{d}^{-1} h(\bar{d}, \beta)}{\beta}\left[\beta-1+\frac{(1-h(\bar{d}, \beta))}{\bar{g}+1}\right] .
$$

Since all quantities on the right-hand side (RHS) of the above result are positive, i.e. $\frac{n \bar{d}^{-1} h(\bar{d}, \beta)}{\beta}>0$, it implies, based on (A2), that

$$
-\frac{\partial h(\bar{d}, \beta)}{\partial \bar{d}}>0
$$

requires the following condition to be satisfied

$$
\left[\beta-1+\frac{(1-h(\bar{d}, \beta))}{\bar{g}+1}\right]>0, \forall \bar{d} \in(0,0.5] .
$$

By inserting $h(\bar{d}, \beta)=\frac{\bar{g}}{(1+\bar{g})^{\frac{1}{\beta}}-1}$ in (A3), the latter can be rewritten as

$$
\left(1+\bar{g}\left(\frac{\beta-1}{\beta}\right)\right)^{\beta}>(1+\bar{g})^{\beta-1},
$$

which is always true since $\bar{g}>0$ and $\beta \geqslant 1$. Hence, based on all the previous equations, $\frac{\partial f(\bar{d}, \theta)}{\partial d}>0$ or in other words, $f$ is a strictly increasing function of $\bar{d}$. 
Consequently, if $h(\bar{d})$ is always larger than $(1+\bar{G}(\bar{d}, \theta))$, then $f(\vec{d}, \theta)$ will have no root and coordinated allocation will always achieve better EE performance than orthogonal allocation. Given that $-h(\bar{d})$ is an increasing function, the minimum value of $h(\bar{d})$ occurs at $\bar{d}=0.5$. Whereas the maximum value of $\bar{G}(\bar{d}, \theta)$ also occurs at $\bar{d}=0.5$. If the minimum value of $h(\bar{d})$ is larger than the maximum value of $\bar{G}(\bar{d}, \theta)$, it implies that the boundary would not exist since the gain function would always be negative. Otherwise, a boundary could exist. Therefore, a necessary and sufficient condition for $f(\bar{d}, \theta)$ to have a unique root over $\bar{d} \in(0.0 .5], \quad \theta \in\left[0, \frac{2 \pi}{3}\right]$ when $n \geqslant 1$ and $A \leqslant 100$ is

$$
h(0.5) \leqslant(1+\max \bar{G}(0.5, \theta)) .
$$

\section{APPENDIX B}

\section{PROOF OF PROPOSITION 2}

Letting (5a) and (5b) to be equal and knowing that $\bar{g}$ is non-negative, it implies, according to the binomial theorem, that

$$
\bar{g}^{\beta-1}+\beta \bar{g}^{\beta-2}(1+\bar{G})+\cdots+(1+\bar{G})^{\beta-1}((\beta-1)-\bar{G})=0,
$$

when $\beta$ is an integer. Given that $\beta \in(1, M]$, considering a three-sector layout, i.e. $M=3$, the above equation becomes a quadratic function, such that

$$
\bar{g}=\frac{1+\bar{G}}{2}(-3+\sqrt{1+4 \bar{G}}),
$$

when $\beta=M$. Given that $\bar{g}>0$, there exists a unique $\bar{G}>0$, such that $\bar{g}=\frac{1+\bar{G}}{2}(-3+\sqrt{1+4 \bar{G}})$. Recall that $\bar{g}(\bar{d})=A \bar{d}^{-n}$ according to the pathloss model, hence finding an upper bound for $\bar{g}(\bar{d})$, is equivalent to finding a lower bound for $D$.

As it is proved in Appendix A that

$$
\frac{\partial \bar{G}(\bar{d}, \theta)}{\partial \bar{d}} \geqslant 0, \forall \theta \in\left[0, \frac{\pi}{3}\right], \forall \bar{d} \in(0,0.5],
$$

the maximum value of $\bar{G}(\bar{d}, \theta)$ is equal to $\max _{\theta} \bar{G}(0.5, \theta)$, which is

$$
\max _{\bar{d}, \theta} \bar{G}(\bar{d}, \theta)=\max _{\theta} \bar{G}(0.5, \theta) \geqslant \bar{G}\left(0.5, \frac{\pi}{3}\right),
$$

where $\bar{G}\left(0.5, \frac{\pi}{3}\right)=A 2^{n}\left(1+3^{-\frac{n}{2}}\right)$. In fact, numerical analysis shows that

$$
\max _{\theta} \bar{G}(0.5, \theta) \leqslant 1+A 2^{n}\left(1+3^{-\frac{n}{2}}\right) .
$$

Consequently,

$$
\bar{g}_{\text {max }}=\frac{1+\max _{\theta} \bar{G}(\bar{d}, \theta)}{2}\left[-3+\sqrt{1+4 \max _{\theta} \bar{G}(\bar{d}, \theta)}\right],
$$

by substituting $\max _{\theta} \bar{G}(0.5, \theta)$ with $1+A 2^{n}\left(1+3^{-\frac{n}{2}}\right)$ in the above equation and rearranging it according to $\bar{g}(\bar{d})=A \bar{d}^{-n}$, the lower bound for the boundary can be obtained by

$D \geqslant\left(\frac{2 A}{\left(2+A 2^{n}\left(1+3^{-\frac{n}{2}}\right)\right)\left[-3+\sqrt{5+A 2^{n+2}\left(1+3^{-\frac{n}{2}}\right)}\right]^{+}}\right)^{\frac{1}{n}}$

and $[X]^{+} \triangleq \max \{x, 0\}$.

Given $f(\bar{d}, \theta, \beta)$ is an increasing function over $\bar{d}$, in Case 2 of Corollary 1 , if both $f(0, \theta, \beta)$ and $f(\underline{D}, \theta, \beta)$ are negative, it indicates that $f(\bar{d}, \theta, \beta)$ has one and only one root $D$ and the lower bound, $\underline{D}$, when $M=3$ is also the lower bound for $\beta \in(1, M)$. While in Case 3 of Corollary 1 , if $f(0, \theta, \beta)$ is negative and $f(\underline{D}, \theta, \beta)$ is positive, it indicates that $f(\bar{d}, \theta, \beta)$ has one and only one root $D$ and the lower bound, $\underline{D}$, when $M=3$ is the upper bound for $\beta \in(1, M)$.

\section{REFERENCES}

[1] J. G. Andrews, S. Buzzi, W. Choi, S. V. Hanly, A. Lozano, A. C. K. Soong, and J. C. Zhang, "What will 5G be?" IEEE J. Sel. Areas Commun., vol. 32, no. 6, pp. 1065-1082, 2014.

[2] S. Buzzi, C. L. I, T. E. Klein, H. V. Poor, C. Yang, and A. Zappone, "A survey of energy-efficient techniques for $5 \mathrm{~g}$ networks and challenges ahead," IEEE Journal on Selected Areas in Communications, vol. 34, no. 4, pp. 697-709, April 2016.

[3] A. Fehske, G. Fettweis, J. Malmodin, and G. Biczok, "The global footprint of mobile communications: The ecological and economic perspective," vol. 49, no. 8, 2011.

[4] G. Auer, V. Giannini, C. Desset, I. Godor, P. Skillermark, M. Olsson, M. Imran, D. Sabella, M. Gonzalez, O. Blume, and A. Fehske, "How much energy is needed to run a wireless network?" vol. 18, no. 5 , pp 40-49, 2011.

[5] J. Hoydis, M. Kobayashi, and M. Debbah, "Green small-cell networks," Vehicular Technology Magazine, IEEE, 2011.

[6] Y. Chen, S. Zhang, S. Xu, and G. Y. Li, "Fundamental trade-offs on green wireless networks," vol. 49, no. 6, pp. 30-37, 2011.

[7] D. Feng, C. Jiang, G. Lim, J. Cimini, L.J., G. Feng, and G. Li, "A survey of energy-efficient wireless communications," IEEE Commun. Surveys Tuts., vol. 15, no. 1, pp. 167-178, 2013.

[8] G. Li, Z. Xu, C. Xiong, C. Yang, S. Zhang, Y. Chen, and S. Xu, "Energyefficient wireless communications: tutorial, survey, and open issues," vol. 18, no. 6, pp. 28-35, 2011.

[9] T. Yang, F. Heliot, and C. H. Foh, "A survey of green scheduling schemes for homogeneous and heterogeneous cellular networks," IEEE Commun. Mag., vol. 53, no. 11, pp. 175-181, 2015.

[10] G. Fettweis and E. Zimmermann, "ICT energy consumption-trends and challenges," in Proc. 11th Int. Symp. on WPMC, 2008.

[11] R. G. Gallager, Information Theory and Reliable Communication. John Wiley \& Sons, Inc., 1968.

[12] F. Héliot, M. A. Imran, and R. Tafazolli, "Energy-efficient resource allocation for orthogonal multi-antenna multi-carrier channel," in Proc. IEEE OnlineGreenComm, Online, Oct. 2013.

[13] D. W. K. Ng, E. S. Lo, and R. Schober, "Energy-efficient resource allocation in multi-cell OFDMA systems with limited backhaul capacity," vol. 11, no. 10, pp. 3618-3631, 2012.

[14] L. Venturino, A. Zappone, C. Risi, and S. Buzzi, "Energy-efficient scheduling and power allocation in downlink OFDMA networks with base station coordination," vol. 14, no. 1, pp. 1-14, 2015.

[15] F. Héliot, M. Imran, and R. Tafazolli, "Low-complexity energy-efficient resource allocation for the downlink of cellular systems," vol. 61 , no. 6 , pp. 2271-2281, 2013.

[16] J. Jiang, M. Peng, K. Zhang, and L. Li, "Energy-efficient resource allocation in heterogeneous network with cross-tier interference constraint," in Proc. IEEE 24th Int. Symp. on PIMRC Workshops, 2013.

[17] Z. Zhang, H. Zhang, Z. Zhao, H. Liu, X. Wen, and W. Jing, "Low complexity energy-efficient resource allocation in down-link dense femtocell networks," in Proc. IEEE 24th Int. Symp. on PIMRC, 2013.

[18] X. Xiao, X. Tao, Y. Jia, and J. Lu, "An energy-efficient hybrid structure with resource allocation in OFDMA networks," in Proc. IEEE WCNC, 2011.

[19] D. Gesbert and M. S. Alouini, "How much feedback is multi-user diversity really worth?" in Communications, 2004 IEEE International Conference on, vol. 1, June 2004, pp. 234-238.

[20] R. V. Rasmussen and M. A. Trick, "Round robin scheduling-a survey," European Journal of Operational Research, vol. 188, no. 3, pp. 617-636, 2008.

[21] G. Miao, N. Himayat, G. Li, and S. Talwar, "Low-complexity energyefficient scheduling for uplink OFDMA," vol. 60 , no. 1, pp. 112-120, 2012.

[22] T. Novlan, J. G. Andrews, I. Sohn, R. K. Ganti, and A. Ghosh, "Comparison of fractional frequency reuse approaches in the OFDMA cellular downlink," in IEEE GLOBECOM, 2010.

[23] M. Rahman and H. Yanikomeroglu, "Enhancing cell-edge performance: a downlink dynamic interference avoidance scheme with inter-cell coordination," IEEE Trans. Wireless Commun., vol. 9, no. 4, pp. 14141425,2010

[24] T. Q. Quek, Z. Lei, and S. Sun, "Adaptive interference coordination in multi-cell OFDMA systems," in IEEE International Symposium on Personal, Indoor and Mobile Radio Communications. IEEE, 2009, pp. 2380-2384.

[25] C. C. Coskun, K. Davaslioglu, and E. Ayanoglu, "Three-stage resource allocation algorithm for energy-efficient heterogeneous networks," IEEE Transactions on Vehicular Technology, vol. PP, no. 99, pp. 1-1, 2017. 
[26] T. Yang, F. Heliot, and C. H. Foh, "Boundary-enabled fair scheduling in downlink multi-carrier multiple-access networks," in IEEE ISCC, 2017

[27] L. Zhou, D. Wu, J. Chen, and Z. Dong, "Greening the smart cities: Energy-efficient massive content delivery via $\mathrm{d} 2 \mathrm{~d}$ communications," IEEE Transactions on Industrial Informatics, vol. 14, no. 4, pp. 1626-1634, April 2018.

[28] D. Wu, L. Zhou, Y. Cai, and Y. Qian, "Collaborative caching and matching for d2d content sharing," IEEE Wireless Communications, vol. 25, no. 3, pp. 43-49, JUNE 2018.

[29] J. M. Meredith, "Study on downlink multiuser superposition transmission for LTE," TSG RAN Meeting 67 RP-75()490, Tech. Rep., March 2015.

[30] M. K. Karakayali, G. J. Foschini, and R. A. Valenzuela, "Network coordination for spectrally efficient communications in cellular systems," IEEE Wireless Commun., vol. 13, no. 4, pp. 56-61, 2006.

[31] S. Das, H. Viswanathan, and G. Rittenhouse, "Dynamic load balancing through coordinated scheduling in packet data systems," in IEEE INFOCOM, vol. 1, March 2003, pp. 786-796 vol.1.

[32] L. Venturino, N. Prasad, and X. Wang, "Coordinated scheduling and power allocation in downlink multicell OFDMA networks," vol. 58, no. 6, pp. 2835-2848, 2009.

[33] P. Viswanath and D. N. C. Tse, "Sum Capacity of the Vector Gaussian Broadcast Channel and Uplink-Downlink Duality," vol. 49, no. 8, pp. 1912-1921, 2003.

[34] S. Verdu, "Spectral Efficiency in the Wideband Regime," vol. 48, no. 6, pp. 1319-1343, 2002

[35] S. R. D. Gonzalez, M. Garcia-Lozano and J. Olmos, "Static intercell interference coordination techniques for LTE networks: A fair performance assessment," in MACOM, 2010.

[36] A. S. Hamza, S. S. Khalifa, H. S. Hamza, and K. Elsayed, "A survey on inter-cell interference coordination techniques in OFDMA-based cellular networks," IEEE Communications Surveys Tutorials, vol. 15, no. 4, pp. 1642-1670, Fourth 2013

[37] F. Héliot, T. Yang, and C. Foh., "Low-complexity green scheduling for the downlink of coordinated cellular system," in IEEE ICC, 2015.

[38] F. Héliot, T. Yang, and C. Foh, "Low-complexity green scheduling for the coordinated downlink of hetnet system," in IEEE CAMAD, 2015.

[39] P. Cao, Z. Chong, Z. Ho, and E. Jorswieck, "Energy-efficient power allocation for amplify-and-forward mimo relay channel," in IEEE 17th International Workshop on CAMAD, 2012.

[40] A. Ambrosy, G. Auer, O. Blume, M. Caretti et al., "D2. 2: definition and parameterization of reference systems and scenarios," INFSOICT-247733 EARTH (Energy Aware Radio and NeTwork TecHnologies) Tech. Rep., 2010.

[41] G. Miao, N. Himayat, and G. Y. Li, "Energy-Efficient Link Adaptation in Frequency-Selective Channels," vol. 58, no. 2, pp. 545-554, Feb. 2010. 